\title{
Simulation and Performance Analysis of Communication System Based on OFDM
}

\author{
J. Cai, L. Wang, Y.Z. Wang \\ Hunan Normal University \\ Physics and Information Science College \\ Hunan, China
}

\begin{abstract}
In order to study the performance of Orthogonal Frequency Division Multiplexing (OFDM) communication system, this article establishes two communication system models of OFDM, which are simulated with MATLAB. Based on this, simulation curves of OFDM performance on different channel models and channel estimation algorithms are obtained and analyzed. It is obvious that among four kinds of European Cooperation in the Field of Scientific and Technical Research Rural 207 channel models, also known as Rural Area (RA), Bad Urban (BU), Typical Urban (TU) and Hilly Terrain (HT), the HT model is the most sensitive to Doppler frequency shift, and the TU model is the least. Simplification of Singular Value Decomposition (SVD) algorithm for the system is only suitable for the low SNR condition. The article offers a reference that the overall performance of the system is improved by correctly and quickly choosing suitable channel estimation algorithm on different channels.
\end{abstract}

Keywords-OFDM; channel estimation; performance simulation

\section{INTRODUCTION}

As a special multi-carrier modulation scheme, Orthogonal Frequency Division Multiplexing (OFDM) has drawn great attention due to its high spectral efficiency and its robustness in multi-path fading and narrowband interference.

An integrated OFDM communication link in Figure 1 consists of modules such as modulation, demodulation, channel estimation, channel model, channel coding, cyclic prefix, and channel decoding, etc. Analysis and simulation results can be found in significant numbers of articles focusing on the effect of the above modules on the performance of OFDM system. The influence of modulation on OFDM system is demonstrated by L.H. Zhang in [1]. He investigated the influence of SNR and the Doppler frequency shift on BER on OFDM in 6-path Rayleigh fading channel, but the investigation was performed under the condition that the influence of multi-path fading on BER was neglected. A.Q. Lv, etc, analyzed the influence on OFDM performance caused by the cyclic prefix (CP), and two different channel estimation algorithms which are least squares (LS) and Linear Minimum Mean-Square Error (LMMSE) (see, e.g., [2]). The analysis and comparison on these two channel estimation algorithms were given by J.K. Zhang, etc, in [3]. LMMSE is complicated, but unfortunately neither A.Q. Lv nor J.K. Zhang paid their attention on the simplified LMMSE algorithm. J.Z. Zeng, etc, analyzed and simulated LS, LMMSE and low rank simplified Singular Value Decomposition (SVD) algorithms in [4], but the influence of channel estimation module on system performance was not covered in this article.

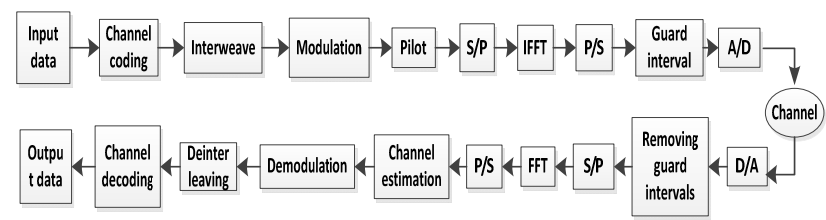

FIGURE I. OFDM SYSTEM SIMULATION LINK

\section{ORTHOGONAL FREQUENCY DiVISION MULTIPLEXING} (OFDM)

\section{A. The Basic Principle of OFDM}

OFDM is a multi-carrier transmission scheme with high spectrum efficiency. The information flow is de-serialized into N-path parallel low rate sub data stream. Assume that the clock cycle of sub data stream is Ts, then the clock cycle of parallel signal after de-serialization $\mathrm{T}=\mathrm{NTs}$. Sub data stream is modulated by corresponding sub-carriers. The frequency of each sub-carrier can be expressed by

$$
f_{n}=f_{0}+n /\left(N T_{S}\right), n=0,1, \cdots, N-1
$$

where $f_{0}$ is intermediate frequency. It can be seen that the subcarriers are mathematically orthogonal. Assume that $\left[d_{0}, \ldots, d_{N-1}\right]$ indicates the sequence of data symbols. The transmitted signal is

$$
S=\sum_{n=0}^{N-1} d_{n} e^{j 2 \pi f_{n} t}=e^{j 2 \pi f_{0}} \sum_{n=0}^{N-1} d_{n} e^{j 2 \pi \frac{n}{N T_{S}} t}
$$

Sampled by $\mathrm{t}=m T s \quad(m=0,1, \ldots, N-1)$,we can get $\mathrm{N}$ sample values

$$
S_{\mathrm{m}}=e^{\mathrm{j} 2 \pi \pi_{0}} \sum_{\mathrm{n}=0}^{\mathrm{N}-1} d_{\mathrm{n}} e^{\mathrm{j} 2 \pi \frac{\mathrm{mm}}{\mathrm{N}}},(m=0,1, \ldots, N-1)
$$

Figure 2 and Figure 3 show the OFDM modulation and demodulation principle respectively. Complex signals $C_{n, 0} \cdots$ $C_{n, N-1}$ are data bits to be passed through the digital modulation (such as MPSK, MQAM, etc). The modulation and demodulation of subcarriers can be accomplished by inputting them to the $\mathrm{N}$ point inverse fast Fourier transform (IFFT) and fast Fourier transform (FFT) respectively. 


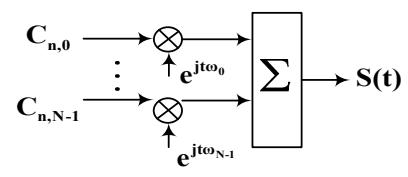

FIGURE II. OFDM MODULATION DEMODULATION

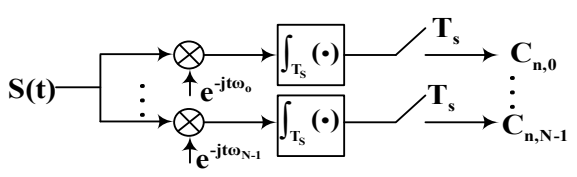

FIGURE III. OFDM

\section{CHANNEL ESTIMATION}

According to the different prior information, channel estimation can be roughly divided into pilot signal estimation and blind estimation. As pilot aided channel estimation is of high estimation accuracy, and can effectively compensate the influence of wireless multi-path fading, it is widely used in applications.

\section{A. Analysis of Channel Estimation Algorithm}

Here briefly introduces two kinds of channel estimation methods based on pilot. One is LS, the other is the LMMSE channel estimation.

LS channel estimation is formulated by

$$
\hat{H}_{p, l s}=X_{p}^{-1} Y_{p}=\left\lfloor\frac{Y_{p}(0)}{X_{p}(0)} \frac{Y_{p}(1)}{X_{p}(1)} \ldots \frac{Y_{p}\left(N_{p}-1\right)}{X_{p}\left(N_{p}-1\right)}\right\rfloor^{T}
$$

where $X_{p}$ is the pilot symbols of the transmitter, $Y_{p}$ is the pilot symbols of the receiver.

The performance of LS channel estimator is not very good because it does not take the influence of the noise into account. but in the premise of ensuring a certain BER, its complexity is relatively low.

The LMMSE channel estimation can be expressed as

$$
\hat{H}_{p, l m m s e}=R_{H_{p} H_{p}}\left(R_{H_{p} H_{p}}+\sigma_{n}^{2}\left(X_{p} X_{p}^{H}\right)^{-1}\right) \hat{H}_{p, l s}
$$

where $\hat{H}_{p, l s}$ is LS estimation value of $H_{p}, R_{H р H p}$ is the autocovariance matrix of channel vector, $\sigma_{n}^{2}$ is the variance of the white Gaussian noise signal. The MMSE estimator is simplified as

$$
\hat{H}_{p}=R_{H_{p} H_{p}}\left(R_{H_{p} H_{p}}+\frac{\beta}{S N R} I\right)^{-1} \hat{H}_{p, l s}
$$

where $S N R=E X_{p}(\kappa)^{2} / \sigma_{n}^{2}$ represents the average signal-to-noise ratio. In this article, the value is taken as $1 ; \beta=E X_{p}(\kappa)^{2} E 1 / X_{p}(\kappa)^{2}$ is a constant only depends on the signal constellation diagram.and it is taken to be 17/9 in this article. Based on SVD method, the simplified estimator is

$$
\hat{H}_{p}=U\left[\begin{array}{cc}
\Delta_{p} & 0 \\
0 & 0
\end{array}\right] U^{H} \hat{H}_{L S}
$$

where $R_{H_{p} H_{p}}=U \Lambda U^{H}, \Lambda$ is a diagonal matrix, of which the diagonal entries are the $\mathrm{N}$ characteristic values of $R_{H_{p} H_{p}}$ distributed in decreasing order. In order to simplify the calculation, we only consider the first $\mathrm{P}$ characteristic values in $\Lambda$. The rest N-P characteristic values are set to zero. P is set to 16 during simulation.

\section{The Simulation Results}

Simulations are performed according to the configurations in Table 1 using un-coded OFDM system. The simulation

\begin{tabular}{|c|c|c|c|}
\hline & modulation & Channel model & $\begin{array}{l}\text { Channel } \\
\text { estimation }\end{array}$ \\
\hline Fig 4 & QDPSK & $\begin{array}{l}1 \text { Gauss } \\
2 \text { 2path Rayleigh+Gauss } \\
3 \text { 4path Rayleigh+Gauss } \\
4 \text { 6path Rayleigh+Gauss }\end{array}$ & LMS \\
\hline Fig 5 & QDPSK & 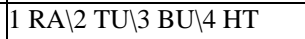 & LMS \\
\hline Fig 6 & 16_QAM & $\begin{array}{l}\text { Multi-path } \quad \text { Rayleigh+ } \\
\text { Gauss }\end{array}$ & $\begin{array}{l}1 / 2 \mathrm{LS} \\
3 \text { LMMSE } \\
4 \text { SVD } \\
\end{array}$ \\
\hline
\end{tabular}
results are shown in Figure 4, Figure 5 and Figure 6.

TABLE I. SIMULATION PARAMETERS

\begin{tabular}{|l|l|l|l|l|l|}
\hline & $\begin{array}{c}\text { Sub- } \\
\text { carrier }\end{array}$ & B/symbol & CP & FFT & $\begin{array}{c}\text { Symbol/car } \\
\text { rier }\end{array}$ \\
\hline Fig 4 & 200 & 2 & $\mathrm{~T} / 4$ & 1024 & 50 \\
\hline Fig 5 & 200 & 2 & $\mathrm{~T} / 4$ & 1024 & 50 \\
\hline Fig 6 & 256 & 4 & 16 & 128 & 100 \\
\hline
\end{tabular}

Figure 4 shows the BER curve of system in the Gaussian channel and multi-path Rayleigh fading channel. When SNR is zero, the BER of OFDM is about 0.2 in every channel. When SNR is 10, the BER of OFDM is 0.0002, 0.0023, 0.0045, 0.015 in Gaussian, 2-path, 4-path and 6-path channels respectively. This shows that the performance of the system improves as the SNR rises. But the improving effect falls down as the quantity of the paths increases, because the more paths, the more serious channel delay spread is. If SNR is 10, every time when two more paths are added to the channel, the error rate decrease $0.0023,0.002,0.0105$ respectively. It is obvious that the relationship between BER and path quantity is non-linear.

Figure 5 shows the BER curve of OFDM system in COST 207 channel. It can be seen that as the velocity of the mobile station getting faster, the Doppler frequency shift $f_{d(\max )}$ becomes greater, the BER climbs as well, so the system performance deteriorates. This is because the Doppler frequency shift will destroy the orthogonality among subcarriers, which will result in inter sub-carrier interference, therefore affect the system performance.We usually take diversity or equalization techniques, etc, to improve the performance of the system. In the same Doppler frequency shift, the difference of BER between different channels is about $0 \sim 0.1$. With the increase of the Doppler frequency shift, the range of difference is narrowed. When $f_{d(\max )}<250 \mathrm{~Hz}$, the BER of HT is the lowest. The BER of the system is 
$\mathrm{HT}<\mathrm{TU}<\mathrm{RA}<\mathrm{BU}$. When $f_{d(\max )}>250 \mathrm{~Hz}$, the BER of HT is the highest, the BER of the system is $\mathrm{RA}<\mathrm{BU}<\mathrm{TU}<\mathrm{HT}$. The HT channel is greatly influenced by Doppler frequency shift, the TU channel minimal influenced.

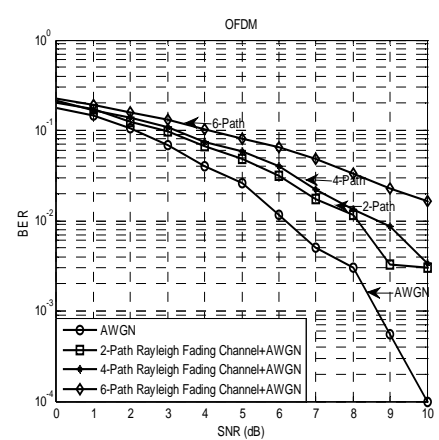

FIGURE IV. PERFORMANCE CURVES OF OFDM IN GAUSS AND MULTIPATH RAYLEIGH FADING CHANNEL

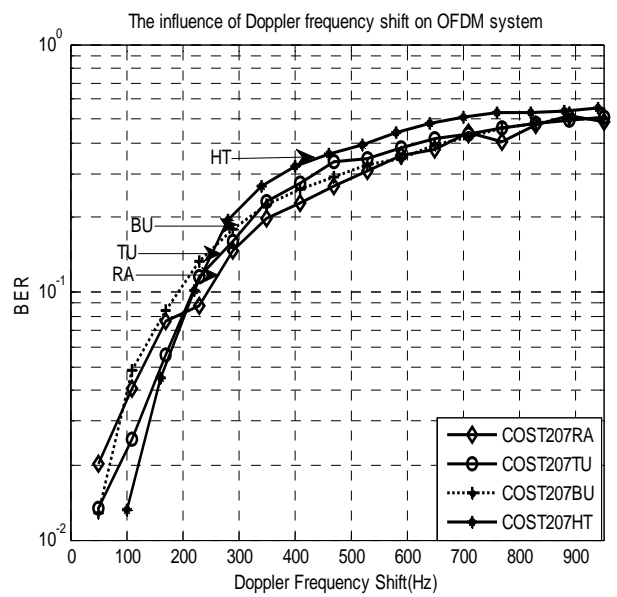

FIGURE V. PERFORMANCE CURVE OF OFDM IN COST 207 CHANNEL MODEL

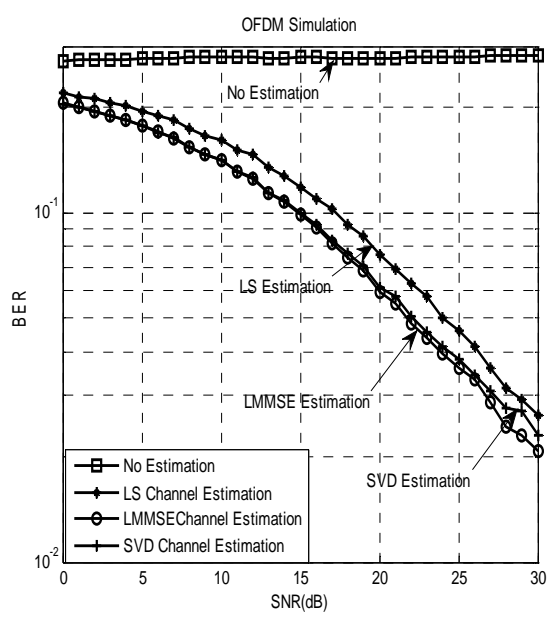

FIGURE VI. PERFORMANCE CURVES OF OFDM IN DIFFERENT CHANNEL ESTIMATION
A frame of OFDM symbol block is estimated multiple times using LS, LMMSE and SVD channel estimation algorithms under different SNR conditions. The average BER curve obtained is shown in Figure 6.

It is illustrated in Figure 6 that under the same SNR condition, the BER is considerably decreased using channel estimation. This shows that channel estimation can improve the performance of the system. When SNR is zero, the improvement of the system under channel estimation is about 0.04. When SNR is 22, the improvement of the system under channel estimation is about 0.14 . The performance increases more than three times. This shows that the higher the SNR, the lower the algorithm is influenced by noise, the more obvious channel estimation improves the system performance. In Figure 6 , it can be seen that LMMSE algorithm is slightly superior to LS algorithm in low BER, with the maximum difference about 0.01 . LMMSE can slightly improve the system performance at the expense of much higher complexity. So LS algorithm is preferable when the BER is acceptable, due to its lower complexity and operation cost. When SNR $<14$, the performance of the LMMSE and SVD algorithm is the same. When SNR>14, the LMMSE algorithm is better than the SVD algorithm. This shows that when SNR increases, the performance of SVD algorithm is instable. The simplification of the system by using SVD algorithm is only adaptable at low SNR.

\section{CONCLUSION}

In this article, a complete OFDM system is programmed using MATLAB and computer simulations are conducted to research the influence of multi-path and Doppler frequency shift to the performance of OFDM systems. And, we simulate the OFDM system using three different channel estimation algorithms and compare the performance. We draw the following three conclusions.

(1) OFDM system can effectively combat multi-path effect, but the multi-path effect still has influence on the performance of OFDM system. The more paths, the more serious delay, the worse performance of the system. BER and the paths is nonlinearly related.

(2) The performance of OFDM is influenced by Doppler frequency shift. As the frequency shift gets larger, the performance of the system becomes worse. Among RA, BU, TU and HT channel models, the HT model is most sensitive to Doppler frequency shift, and the TU model is the least.

(3) In OFDM system, the higher the SNR, the more obviously channel estimation improves the system performance. LS and LMMSE are two kinds of channel estimation algorithms. The simulation results verify the performance of the LMMSE is superior to LS, but the effects are not obvious. In general, the preferred choice is LS algorithm. When SNR increases, the performance of the SVD algorithm is not stable. Its simplification to the system is only suitable under low SNR conditions.

\section{REFERENCES}

[1] L.H. Zhang, G.H. Kang. Analysis of the simulation performance of OFDM system in multipath fading channels [J]. computer simulation, 24 (7): 130-133, 2007. 
[2] A.Q. Lv, Y.M. Tian, M.H. Zhu. Simulation and performance analysis of OFDM system based on the MATLAB [J]. computer simulation, 22 (10): 164-168, 2005.

[3] J.K. Zhang, X.M. Mu, E.Q. Chen, et al. The algorithm Analysis of channel estimation for OFDM system based on pilot [J]. communication technology, 42 (08): 91-98, 2009.

[4] J.Z. Ceng, H.Q. Huang. The study of performance of the channel estimation algorithm based on the pilot for OFDM system [J]. communication technology,43 (10): 54-56, 2010. 